\title{
A review of Caesarean section techniques and postoperative thromboprophylaxis at a tertiary hospital
}

\author{
Chang Qi Hester $\underline{\text { Lau }}^{1}$, MBBS, Tuck Chin Tiffany Wong ${ }^{1}$, MBBS, Eng Loy $\underline{\text { Tan }}^{2}$, MMed, MRCoG, \\ Devendra Kanagalingam ${ }^{2}$, MBBS, FRCOG
}

INTRODUCTION Although Caesarean sections (CSs) are among the most commonly undertaken procedures in the world, there are wide variations in the surgical techniques used. This study aimed to: (a) review the surgical techniques used for CS by obstetricians working in a tertiary hospital in Singapore; (b) compare the techniques with those recommended in evidence-based guidelines; and (c) examine the relationship between the technique used and the level of seniority of the surgeons.

METHODS Data on 490 CSs performed in Singapore General Hospital (SGH) between 1 August 2013 and 30 June 2014 was collected from the Delivery Suite database and reviewed. The surgical techniques studied were closure of the pelvic and parietal peritoneum, closure of the uterine layer, use of surgical drains and use of postoperative thromboprophylaxis. RESULTS A total of 486 CSs were analysed after four cases were excluded due to missing data. Most fetal head deliveries were manual. The majority of surgeons did not close the peritoneum; most of those who did were senior surgeons. Double-layer uterine closures were done for all cases and drain usage was rare. $2.0 \%$ of the patients received grossly inadequate thromboprophylaxis.

CONCLUSION The surgical techniques currently practised in SGH are closely aligned with those of the evidence-based guidelines. Peritoneal closure appears to be associated with the surgeon's early training, with a greater number of senior surgeons being less willing to abandon this step. Greater vigilance in implementing appropriate thromboprophylaxis is recommended.

Keywords: Caesarean techniques, LSCS, peritoneal closure, pregnancy, thromboprophylaxis

\section{INTRODUCTION}

Caesarean sections (CSs) are one of the most commonly undertaken procedures in the world. At Singapore General Hospital (SGH), Singapore, CS rates increased from $17.8 \%$ in 1999 to $34.0 \%$ in 2009. ${ }^{(1)}$ Common indications for CS include fetal distress, poor progress in labour and malpresentations.

Although the procedure is commonly performed, CS techniques are highly variable, and are usually dependent on the surgeon and the urgency of the procedure. ${ }^{(2)}$ In a study conducted by Tully et al, ${ }^{(2)}$ a survey was sent to all members and fellows of the Royal College of Obstetrician and Gynaecologists (RCOG) residing in the United Kingdom (UK) to gain insight into their surgical approach toward both elective and emergency CS. Tully et al found that only a few techniques (i.e. double closure of the uterus, use of prophylactic antibiotics and use of a lower transverse skin incision for abdominal entry) were utilised by more than $80 \%$ of the obstetricians. ${ }^{(2)}$

In 2011, the UK's National Institute for Health and Clinical Excellence (NICE) published recommendations for specific steps used in $\mathrm{CS}$, with the aim of reducing the morbidity rate of the procedure. ${ }^{(3)}$ The following are some of NICE's recommendations: (a) closure of the uterus - as the effectiveness and safety of single-layer closure of uterine incision is uncertain, the uterine incision should be sutured in two layers, except within a research context; (b) closure of the peritoneum - neither the visceral nor parietal peritoneum should be sutured in CS, because non-closure reduces operating time and the need for postoperative analgesia, and improves maternal satisfaction; and (c) use of superficial wound drains - they should not be routinely used in CS because superficial wound drains do not decrease the incidence of wound infection or wound haematoma.

Many of the surgical techniques used in CS have not undergone rigorous assessment; in fact, only a few randomised controlled trials have evaluated CS techniques. A Cochrane review of single-layer vs. double-layer uterine incision closure, involving 19 randomised controlled trials, concluded that there was no statistical difference between the two types of uterine incision closure in terms of the primary outcome of postoperative febrile morbidity and secondary maternal outcomes (i.e. blood loss and risk of blood transfusion). ${ }^{(4)}$ Another Cochrane review examined the outcomes of the closure of both peritoneal layers vs. the non-closure of both or only one peritoneal layer, and found that non-closure of both peritoneal layers resulted in shorter operative times and shorter postoperative hospitalisation duration, except when compared to closure of only the visceral layer. ${ }^{(5)}$ Both of the aforementioned Cochrane reviews ${ }^{(4,5)}$ included the CAESAR study and the CORONIS study, which are both large randomised controlled trials. In the CAESAR study, ${ }^{(6)}$ it was shown that single-layer vs. double-layer closure of the uterine incision, closure vs. non-closure of the pelvic peritoneum, and liberal vs. restricted use of a subrectus sheath drain made no significant difference in the risk of maternal infectious morbidity. 
The CORONIS study ${ }^{(7)}$ looked at technique pairs: blunt vs. sharp entry; exterior vs. intra-abdominal repair of the uterus; single-layer vs. double-layer closure of the uterus; closure vs. non-closure of the pelvic and parietal peritoneum; and the use of chromic catgut vs. polyglactin-910 for uterine closure. It found no statistically significant difference within any of the pairs of techniques in terms of the composite of death, maternal infectious morbidity, and the incidence of further operative procedures or blood transfusion up to six weeks postpartum. ${ }^{(7)}$

The use of venous thromboembolism (VTE) prophylaxis after surgery is another important aspect of CS, as VTE has consistently been shown to be the leading cause of maternal mortality and morbidity in the developed world. ${ }^{(8,9)}$ The RCOG evidence-based Green-top Guideline No. 37a, 'Reducing the Risk of Venous Thromboembolism during Pregnancy and the Puerperium', recommended that "All women who have had CSs should be considered for thromboprophylaxis with low-molecular-weight heparin (LMWH) for ten days after delivery apart from low-risk women having an elective CS, who should be considered for thromboprophylaxis with $L M W H$ for ten days after delivery if they have any additional risk factors" ${ }^{\prime 10)}$

The present study aimed to review the surgical techniques used for CS in SGH, a tertiary hospital in Singapore. The CS techniques that were analysed included those that were examined in the CAESAR study and postoperative thromboprophylaxis. The current practices of SGH obstetricians were also compared with the recommendations of evidence-based guidelines. The surgical techniques chosen by different surgeons in relation to their level of seniority were also analysed.

\section{METHODS}

This was a retrospective study involving CS performed in SGH between 1 August 2013 and 30 June 2014. Data on CS techniques employed by the primary surgeon were collected from the CS pro forma system implemented in SGH. This pro forma system ensures that the details of each CS are systematically recorded and stored in a computerised Delivery Suite database. The following techniques were studied: (a) closure of the pelvic and parietal peritoneum; (b) closure of the uterine layer; (c) use of surgical drains; and (d) use of postoperative thromboprophylaxis.

Closure of either the pelvic peritoneum or parietal peritoneum is not recommended. The pelvic peritoneum refers to the layer of peritoneum overlying the uterine incision, while the parietal peritoneum refers to the outer layer of the peritoneum that is attached to the abdominal muscle wall. Uterine layer closure includes single-layer closure and double-layer closure. In singlelayer closure, both edges of the uterine incision are brought together with a single layer of sutures. In double-layer closure, the uterine incision is closed using two layers of sutures; the first layer is usually a full-thickness suture that opposes the endometrial and myometrial layers, while the second layer is an imbricating suture that opposes the layer of myometrium to cover the first layer. The use of a subrectus sheath drain is clinically indicated when haemostasis is not achieved even though appropriate haemostatic procedures have been performed.
Table I. Demographic characteristics of the women $(n=486)$.

\begin{tabular}{|c|c|}
\hline Characteristic & No. (\%) \\
\hline Mean age (yr) & 32.1 \\
\hline \multicolumn{2}{|l|}{ Type of pregnancy } \\
\hline Singleton & $451(92.8)$ \\
\hline Multiple & $35(7.2)$ \\
\hline \multicolumn{2}{|l|}{ Parity } \\
\hline Primiparous & $258(53.1)$ \\
\hline Multiparous & $228(46.9)$ \\
\hline \multicolumn{2}{|l|}{ Previous CS } \\
\hline Yes & $167(34.4)$ \\
\hline No & $319(65.6)$ \\
\hline \multicolumn{2}{|l|}{ Gestation at admission } \\
\hline Term ( $\geq 37$ wk) & $377(77.6)$ \\
\hline Late preterm $\left(35-36^{+6} \mathrm{wk}\right)$ & $50(10.3)$ \\
\hline Early preterm (<35 wk) & $59(12.1)$ \\
\hline \multicolumn{2}{|l|}{ Urgency of CS } \\
\hline Category 1 & $27(5.6)$ \\
\hline Category 2 & $137(28.2)$ \\
\hline Category 3 & $184(37.9)$ \\
\hline Category 4 & $138(28.4)$ \\
\hline \multicolumn{2}{|l|}{ Primary indication for CS } \\
\hline Maternal* & $240(49.4)$ \\
\hline Fetal $^{+}$ & $139(28.6)$ \\
\hline Labour* & $107(22.0)$ \\
\hline \multicolumn{2}{|l|}{ Seniority of primary surgeon } \\
\hline Senior consultant/consultant & $387(79.6)$ \\
\hline Associate consultant/registrar/resident & $99(20.4)$ \\
\hline
\end{tabular}

*Includes pregnancy-related hypertensive disorders, previous lower-segment CS or uterine operation, multiple pregnancies, antepartum haemorrhage, mother's past medical history and mother's request. +Includes non-reassuring fetal status, intrauterine growth retardation, fetal malpresentation, macrosomia, chorioamnionitis and cord prolapse. ‡lncludes cephalopelvic disproportion, failure to progress, failed induction and failed instrumental delivery. CS: Caesarean section

Table II. Number of women who had peritoneal closure during their Caesarean deliveries and the duration of surgery according to the type of peritoneal closure $(n=486)$.

\begin{tabular}{lcccc}
\hline \multirow{2}{*}{$\begin{array}{l}\text { Peritoneum } \\
\text { procedure }\end{array}$} & No. (\%) & \multicolumn{2}{c}{ Duration of surgery (min) } \\
\cline { 3 - 5 } & & Mean & Minimum & Maximum \\
\hline Closure & & & & \\
Both & $21(4.3)$ & 37.4 & 25 & 50 \\
Pelvic only & $9(1.9)$ & 42.2 & 35 & 50 \\
Parietal only & $10(2.1)$ & 41.5 & 30 & 55 \\
Non-closure & $446(91.8)$ & 41.3 & 20 & 195 \\
\hline
\end{tabular}

CS increases the risk of VTE after delivery. Thromboprophylactic methods include mechanical (e.g. thromboembolism-deterrent [TED] stockings and early ambulation) and pharmacological (e.g. LMWH) thromboprophylaxis. Standard departmental protocol recommends the use of $\mathrm{LMWH}$, TED stockings and early ambulation for all women who have undergone a CS, unless they are allergic to $\mathrm{LMWH}$ or are deemed to have an increased risk of major haemorrhage (e.g. patients who had undergone surgeries for placental abruption or placenta praevia, or had postpartum 
Table III. Seniority of the surgeons who performed peritoneal closure during Caesarean deliveries.

\begin{tabular}{lccc}
\hline \multirow{2}{*}{$\begin{array}{l}\text { Peritoneum } \\
\text { procedure }\end{array}$} & \multicolumn{2}{c}{ No. of closures/non-closures (\%) } \\
\cline { 2 - 4 } & $\begin{array}{c}\text { Senior* } \\
(\mathbf{n}=\mathbf{3 8 7})\end{array}$ & $\begin{array}{c}\text { Junior } \\
(\mathbf{n}=\mathbf{9 9 )}\end{array}$ & $\begin{array}{c}\text { Total } \\
(\mathbf{n}=\mathbf{4 8 6})\end{array}$ \\
\hline Closure & $39(10.1)$ & $1(1.0)$ & $40(8.2)$ \\
Both & $21(5.4)$ & $0(0)$ & $21(4.3)$ \\
Pelvic only & $9(2.3)$ & $0(0)$ & $9(1.9)$ \\
Parietal only & $9(2.3)$ & $1(1.0)$ & $10(2.1)$ \\
Non-closure & $348(89.9)$ & $98(99.0)$ & $446(91.8)$ \\
\hline
\end{tabular}

*Includes consultants and senior consultants. Includes residents, registrars and associate consultants.

Table V. Proportion of patients given low-molecular-weight heparin (LMWH) according to the urgency of Caesarean section.

\begin{tabular}{lccc}
\hline $\begin{array}{l}\text { Urgency of } \\
\text { Caesarean section }\end{array}$ & $\begin{array}{c}\text { Notal } \\
(\mathbf{n}=\mathbf{4 8 6})\end{array}$ & $\begin{array}{c}\text { LMWH* } \\
(\mathbf{n}=\mathbf{3 4 3})\end{array}$ & $\begin{array}{c}\text { No LMWH* } \\
(\mathbf{n}=\mathbf{1 4 3})\end{array}$ \\
\cline { 2 - 4 } & $27(5.6)$ & $21(77.8)$ & $6(22.2)$ \\
Category 1 & $137(28.2)$ & $100(73.0)$ & $37(27.0)$ \\
Category 2 & $184(37.9)$ & $123(66.8)$ & $61(33.2)$ \\
Category 3 & $138(28.4)$ & $99(71.7)$ & $39(28.3)$ \\
\hline
\end{tabular}

*Percentages calculated based on total no. of patients in each category.

haemorrhage). The first dose of LMWH is to be instituted 6-12 hours after surgery (i.e. before the patient is discharged). In the present study, the following measures of postoperative thromboprophylaxis were analysed: early ambulation; use of TED stockings; use of $\mathrm{LMWH}$; dose of $\mathrm{LMWH}$ used; and duration of LMWH use post-delivery. The urgency of CS was categorised according to the following RCOG classification: Category 1 urgent, requiring immediate delivery; Category 2 - urgent, requiring delivery within 75 minutes; Category 3 - semi-urgent, requiring early delivery; and Category 4 - non-urgent.

\section{RESULTS}

Within the duration of the study, $490 \mathrm{CSs}$ were recorded. Of these, four were excluded due to inadequate records in the system. The characteristics of the women included in the study $(n=486)$ are shown in Table I. Collectively, the 486 CSs were performed by a total of 44 surgeons; 387 deliveries were performed by 11 senior surgeons (i.e. consultants and senior consultants) and 99 deliveries were performed by 33 junior surgeons (i.e. residents, registrars and associate consultants).

Forceps were not routinely used in fetal head delivery. 85.7\% of the single cephalic presentation deliveries were done manually, while the remaining $14.3 \%$ were done using forceps; none were done using vacuum. All 486 women underwent doublelayer uterine closure instead of single-layer closure. Peritoneal closure was performed for 40 (8.2\%) women. Table II shows the number of women who had peritoneal closure during their Caesarean deliveries and the duration of surgery according to the type of peritoneal closure performed. The minimum duration of surgery was the shortest for patients who had non-closure of both peritoneal layers. When this information was correlated with the level of seniority of the surgeons who performed the surgery
Table IV. Postoperative thromboprophylactic methods employed for the women who underwent Caesarean section $(n=486)$.

\begin{tabular}{lc}
\hline Method & No. (\%) \\
\hline No thromboprophylaxis & $5(1.0)$ \\
Early ambulation only & $2(0.4)$ \\
TED stocking only & $3(0.6)$ \\
Early ambulation and TED stocking only & $133(27.4)$ \\
LMWH only & $7(1.4)$ \\
LMWH and early ambulation only & $7(1.4)$ \\
LMWH and TED stocking only & $6(1.2)$ \\
All (LMWH, TED stocking and early ambulation) & $323(66.5)$ \\
\hline
\end{tabular}

Table VI. Time interval between completion of surgery and administration of the first dose of LMWH among those who received LMWH ( $\mathrm{n}=343$ ).

\begin{tabular}{lc}
\hline Time interval (hr) & No. (\%) \\
\hline$<6$ & $10(2.9)$ \\
$6-12$ & $232(67.6)$ \\
$>12$ & $101(29.4)$ \\
\hline
\end{tabular}

LMWH: low-molecular-weight heparin

(Table III), we found that most of the peritoneal closures were done by senior surgeons. The peritoneal closures performed in the present study were done by eight senior surgeons and one junior doctor, with the junior doctor being responsible for only one parietal peritoneum closure. On further analysis, it was noted that one of the senior consultants routinely closed the peritoneum.

Among the 486 women who underwent CS, a subrectus drain was used only for one patient. This indicates the rare use of subrectus drains in our hospital. Table IV shows the different permutations of thromboprophylactic measures employed for the included patients. None of the women had contraindications to $\mathrm{LMWH}$ and there were no instances of major haemorrhage due to its use during the study period.

Not all the women who had urgent CS (i.e. Categories 1, 2 and 3) were given LMWH postoperatively (Table V). Analysis of the time interval between completion of surgery and administration of the first dose of LMWH (Table VI) showed a mean and median time interval of 10.78 hours and 8.75 hours, respectively.

\section{DISCUSSION}

The NICE guidelines provide evidence-based recommendations for the care of patients before, during and after CS. ${ }^{(3)}$ Although they include recommendations on surgical techniques, there is still considerable variability in the CS techniques used, likely due to personal preferences, differences in training and a lack of robust evidence to support the use of one technique over another.

The NICE guidelines recommend the use of double-layer uterine closure, except in research settings. ${ }^{(3)}$ In the Cochrane review on uterine closure in $\mathrm{CS}^{\left({ }^{(4)}\right.}$ as well as both the $\mathrm{CAESAR}^{(6)}$ and CORONIS studies, ${ }^{(7)}$ no apparent differences in short-term outcomes (e.g. morbidity from infection, pain, need for blood transfusion and hospital readmission) were identified between single- and double-layer uterine closure. However, observational 
data suggested that there was a higher rate of uterine rupture in subsequent pregnancy when single-layer closures were used. ${ }^{(11)}$ The effectiveness and safety of single-layer uterine closure are still uncertain. All the CSs performed in SGH during our study period used double-layer uterine closure, as recommended by the NICE guidelines. ${ }^{(3)}$

A superficial surgical drain was used in only one patient after CS. Even though the reason for its use was not recorded in the database, it was clear from the results of the study that surgical drains are rarely used in our hospital. This is in concordance with the recommendations of the NICE guidelines, ${ }^{(3)}$ which state that superficial wound drains should not be routinely used in CS because they do not decrease the incidence of wound infection or wound haematoma.

Among all the CSs performed in the present study, closure of one or more layers of peritoneum was done in $8.2 \%$ of the cases. While the minimum duration of surgery was the shortest for women who had non-closure of the peritoneum layers, the mean duration of surgery for this group of women was not lower than that for those who had closure of one or both peritoneum layers; in fact, the mean duration was higher for non-closure than for closure of both peritoneum (Table II). This may be attributed to the small sample size of the group that underwent peritoneal closure in our study $(n=40)$. Both the NICE guidelines and the Cochrane review on peritoneal layer closure concluded that peritoneal non-closure reduces operating time and the need for postoperative analgesia, and improves maternal satisfaction without increasing morbidity and mortality. ${ }^{(3,5)}$ Studies have also shown that there is no significant difference in the adhesion rate between closure and non-closure of peritoneal layers. ${ }^{(3,5,12,13)}$ It is interesting to note that the senior surgeons were more likely to perform peritoneal closure than the junior doctors. This may be due to the training of the senior surgeons, which could have taken place before recommendations discouraging peritoneal closure were published.

The 2015 RCOG Green-top Guidelines advocate that all women who undergo CS should be considered for at least ten days of prophylactic LMWH, with the exception of low-risk women who have elective CSs. ${ }^{(10)}$ In $\mathrm{SGH}$, it is the hospital policy that all women who undergo CS be given thromboprophylaxis with $\mathrm{LMWH}$, unless contraindications exist for the use of $\mathrm{LMWH}$. A combination of LMWH, TED stockings and early ambulation is advocated for all women who have undergone a CS. Such a policy has the benefit of simplicity, minimising the risk of omission of thromboprophylaxis among women who are at high risk of developing VTE. Given that the majority $(71.7 \%)$ of women who underwent CS in the present study had emergency/ unscheduled CS (i.e. RCOG urgency Categories 1, 2 and 3), such a policy on thromboprophylaxis may be reasonable and beneficial. We acknowledge that a small number of low-risk women would receive full VTE prophylaxis with such a policy, but the department's view is that the use of LMWH in these women should not carry any significant risk. Furthermore, the criteria used to define 'low risk' can be problematic, given that risk factors are dynamic and may arise during labour. For instance, postpartum haemorrhage and hospital stay prior to delivery are additional risk factors that can cause a seemingly 'low-risk' woman to become a candidate for thromboprophylaxis. Despite the somewhat liberal policy on thromboprophylaxis in our hospital, only $66.5 \%$ of the women in the present study received the full range of thromboprophylaxis measures. Notably, $2.0 \%$ of the women received grossly inadequate thromboprophylaxis, as defined by absence of any thromboprophylactic measures in the form of LMWH, TED stockings or early ambulation (i.e. no thromboprophylaxis, had early ambulation only or had TED stocking only; Table IV).

It has been established that emergency CSs are associated with up to two times the risk of VTE as compared to planned CS. ${ }^{(14-17)}$ The 2011 RCOG Green-top Guidelines recommend that all women who have had emergency CS (i.e. Category 1, 2 or 3) should receive prophylactic $\mathrm{LMWH}$ for ten days postpartum. According to this guideline, only $77.8 \%, 73.0$ and $66.8 \%$ of the patients in Category 1, 2 and 3, respectively, received the recommended $\mathrm{LMWH}$ in the present study (Table V). It is interesting to note that in the updated 2015 RCOG Green-top Guidelines, the categories of emergency CS were no longer indicated; instead, all women undergoing emergency CS and those having elective CS with high risk factors were recommended for VTE prophylaxis. ${ }^{(10)}$ In addition, among the women who received $\mathrm{LMWH}$, around $29.4 \%$ received the dose after the recommended 6-12 hours after the surgery.

To conclude, the present study is an audit of the CS techniques that were employed by obstetricians in $\mathrm{SGH}$. The results showed that the majority of CS techniques and measures practised in our hospital complied with evidence-based recommendations, except for practices on peritoneal closure and postoperative thromboprophylaxis. There is an urgent need for attention to be given to the administration of appropriate thromboprophylaxis to women who have undergone CS, as deficiencies in this area may result in severe maternal morbidity and mortality. We acknowledge that when differing techniques are not shown to have any significant impact on outcomes, surgeons should be given the freedom to choose the techniques that they are most comfortable with.

\section{ACKNOWLEDGEMENT}

The authors thank the staff of the Department of Obstetrics and Gynaecology, Singapore General Hospital, Singapore, for the help offered during data collection.

\section{REFERENCES}

1. Wang CP, Tan WC, Kanagalingam D, Tan HK. Why we do caesars: a comparison of the trends in caesarean section delivery over a decade. Ann Acad Med Singapore $2013 ;$ 42:408-12.

2. Tully L, Gates S, Brocklehurst P, McKenzie-McHarg K, Ayers S. Surgical techniques used during caesarean section operations: results of a national survey of practice in the UK. Eur J Obstet Gynecol Reprod Biol 2002; 102:120-6.

3. National Institute for Health and Care Excellence. Caesarean section: NICE guidelines [CG132] 2011. Available at: https://www.nice.org.uk/guidance/ cg132. Accessed October 10, 2015.

4. Dodd JM, Anderson ER, Gates S, Grivell RM. Surgical techniques for uterine incision and uterine closure at the time of caesarean section. Cochrane Database Syst Rev 2014; 7:CD004732. 
5. Bamigboye AA, Hofmeyr GJ. Closure vs. non-closure of the peritoneum at caesarean section: short- and long-term outcomes. Cochrane Database Syst Rev 2014; 8:CD000163.

6. CAESAR study collaborative group. Caesarean section surgical techniques: a randomised factorial trial (CAESAR). BJOG 2010; 117:1366-76.

7. CORONIS Collaborative Group, Abalos E, Addo V, et al. Caesarean section surgical techniques (CORONIS): a fractional, factorial, unmasked, randomized controlled trial. Lancet 2013; 382:234-48.

8. Knight M, Kenyon S, Brocklehurst $\mathrm{P}$, et al (eds.) on behalf of MBRRACE-UK. Saving Lives, Improving Mothers' Care. Lessons learned to inform future maternity care from the UK and Ireland Confidential Enquiries into Maternal Deaths and Morbidity 2009-2012. Oxford: National Perinatal Epidemiology Unit, University of Oxford, 2014. Available at: https://www.npeu.ox.ac.uk downloads/files/mbrrace-uk/reports/Saving\%20Lives\%20Improving\%20 Mothers\%20Care\%20report\%202014\%20Full.pdf. Accessed April 11, 2015.

9. Chang J, Elam-Evans LD, Berg CJ, et al. Pregnancy-related mortality surveillance-United States, 1991--1999. MMWR Surveill Summ 2003; 52:1-8.

10. Royal College of Obstetricians \& Gynaecologists. Green-top Guideline No. 37 (April 2015). Reducing the Risk of Venous Thromboembolism during Pregnancy and the Puerperium. Available at: https://www.rcog.org.uk/globalassets/ documents/guidelines/gtg-37a.pdf. Accessed November 1, 2015

11. Gyamfi C, Juhasz G, Gyamfi P, Blumenfeld Y, Stone JL. Single- vs. double-layer uterine incision closure and uterine rupture. J Matern Fetal Neonatal Med 2006; 19:639-43.

12. Tabasi Z, Mahdian M, Abedzadeh-Kalahroudi M. Closure or non-closure of peritoneum in cesarean section: outcomes of short-term complication. Arch Trauma Res 2013; 1:176-9.

13. Kapustian V, Anteby EY, Gdalevich M, et al. Effect of closure vs. nonclosure of peritoneum at cesarean section on adhesions: a prospective randomized study. Am J Obstet Gynecol 2012; 206:56.e1-4.

14. Liu S, Liston RM, Joseph KS, et al. Maternal mortality and severe morbidity associated with low-risk planned cesarean delivery vs. planned vaginal delivery at term. CMAJ 2007; 176:455-60.

15. Ros HS, Lichtenstein P, Bellocco R, Petersson G, Cnattingius S. Pulmonary embolism and stroke in relation to pregnancy: how can high-risk women be identified? Am J Obstet Gynecol 2002; 186:198-203.

16. Macklon NS, Greer IA. Venous thromboembolic disease in obstetrics and gynaecology: the Scottish experience. Scott Med J 1996; 41:83-6.

17. Jacobsen AF, Drolsum A, Klow NE, et al. Deep vein thrombosis after elective cesarean section. Thromb Res 2004; 113:283-8. 